\title{
Estudio comparativo entre el desarrollo psicomotor y el estado nutricional en niños de kínder, pertenecientes a un establecimiento municipal y a uno particular de la ciudad de Temuco
}

\author{
Comparative study between psychomotor development and nutritional status of \\ kindergarten children from a municipal and a private educational institution \\ in the city of Temuco, Chile \\ * Maura Sandoval Vallejos, *Alfredo Fernández Ávila, ***Rodrigo Vargas Vitoria, \\ **Cristián Martínez Salazar, **Vanessa Carrasco Alarcón
}

Sandoval, M., Fernández, A., Vargas, R., Martínez, C. \& Carrasco, V. (2017). Estudio comparativo entre el desarrollo psicomotor y el estado nutricional en niños de kínder, pertenecientes a un establecimiento municipal y a uno particular de la ciudad de Temuco. Revista Ciencias de la Actividad Física UCM, $\mathrm{N}^{\circ}$ 18(2) julio-diciembre, 1-8.

\begin{abstract}
RESUMEN
El presente estudio tiene como objetivo determinar si existen diferencias significativas entre el desarrollo psicomotor y el estado nutricional en niños de 4 a 5 años, pertenecientes a un establecimiento educacional municipal y niños pertenecientes a un establecimiento particular. El diseño es no experimental, transversal y correlacional. El enfoque es de tipo cuantitativo/racionalista. La población está compuesta por 54 niños y niñas de kínder. Para evaluar el desarrollo psicomotor se utilizó el test de Jack Capon, adaptación de Sergio Carrasco (1989). Para conseguir el estado nutricional se obtuvo el peso y la talla de la población. Los resultados indican que los estudiantes del colegio particular, presentaron diferencias significativas en el desarrollo psicomotor, frente a los resultados de los de la escuela municipal. Así también los sujetos con sobrepeso u obesidad, categorizados en malnutrición por exceso presentaron un rendimiento motor inferior en comparación con los normopeso y bajo peso y en el ámbito de la imagen corporal a mayor peso es menor el reconocimiento de ella.
\end{abstract}

\section{PALABRAS CLAVE}

Desarrollo psicomotor, estado nutricional, preescolares, test Jack Capon.

\begin{abstract}
The aim of this research is to determine whether there are significant differences between psychomotor development and nutritional status in 4 to 5 year-old children that attend a municipal school and those that attend a private one. The design is non-experimental, cross-sectional and correlational and the approach is quantitative/rationalist. The sample is made-up of 54 boys and girls in kindergarten. To evaluate psychomotor development, the Jack Capone Test, adapted by Sergio Carrasco (1989), was used. With regards to the nutritional status, the sample group's weight and height were obtained. Results revealed that the children from the private institution showed significant differences in psychomotor development compared to the results of the children from the municipal institution. Furthermore, the overweight or obese individuals, classified as suffering from malnutrition due to excessive amounts of food, showed inferior motor performance compared to the children with normal or below average weight. With regards to body image, the higher the weight, the lower the child's own body image.
\end{abstract}

\section{Key words}

Psychomotor development, nutritional condition, children, preschool students, test Jack Capone.

* Magíster Educación Física, Universidad de la Frontera, Chile.

** Departamento de Educación Física Universidad de la Frontera, Chile.

*** Departamento de Ciencias de la Actividad Física Universidad Católica del Maule, Chile. 


\section{INTRODUCCIÓN}

La adquisición de habilidades durante el ciclo vital es lo que se denomina desarrollo. Es consecuencia de procesos cerebrales definidos genéticamente en interacción permanente con el ambiente. El desarrollo normal depende de un componente genético, un período de gestación adecuado y la influencia de factores medioambientales de orden biológico, socio económico y familiar que actúan en la adquisición de diferentes habilidades (Quino \& Barreto, 2015). Si bien, al nacer cada ser humano tiene un potencial de desarrollo determinado congénitamente, su expresión final es resultado de la interacción de la genética con estímulos recibidos desde el entorno familiar, social y comunitario. Probablemente ese sea el motivo por el cual la intervención temprana tiene alto impacto en el pronóstico de los niños (Schonhaut, Schönstedt, Álvarez, Salinas, \& Armijo, 2010). Un buen estado de salud psicofísico durante los años preescolares es esencial para que los niños comiencen la etapa escolar preparados para aprender, más aún considerando que la salud y el desempeño académico tienen un efecto muy importante sobre sus logros futuros. A medida que crecen, aumenta la influencia del medio que los rodea, y las diferencias en el desarrollo psicomotor entre los niños que reciben distintos niveles de estimulación aumentan (Garibottia, Comar, Vasconi, Giannini \& Pittau, 2013).

No hay por qué suponer que todos los niños sanos y activos que acceden al jardín y/o kínder poseen conocimiento y dominio adecuado de su cuerpo. Algunos niños de estas edades pueden presentar determinadas dificultades relacionadas con la coordinación, el control postural, la lateralidad o la estructuración espacio-temporal, que afecten de algún modo a su desarrollo (Monge, 1999).

Actualmente, en Chile existe alta prevalencia de déficit de desarrollo en la población menor de 6 años, concentrándose en la población de nivel socioeconómico bajo. Un estudio realizado por Seguel (2012) a un grupo de 1.025 niños de 0 a 6 años de diversas comunas de Santiago, mostró que un $16 \%$ de ellos presenta déficit en el desarrollo psicomotor en el grupo de menores de 2 años y un $40 \%$ de déficit en el grupo de 2 a 5 años. El desglose de estos resultados muestra que un $14 \%$ estaba en situación de riesgo y un $2 \%$ presentaba retraso en el desarrollo, y en el grupo preescolar existía un $31 \%$ de riesgo y $9 \%$ de retraso. Además, el estudio evidencia que al analizar por áreas de desarrollo, el $0 \%$ de ellos presentaba déficit en el lenguaje; un 30\% en coordinación, y un 17\% en motricidad. Como se menciona anteriormente, la mayoría de los estudios que evalúan el desarrollo psicomotor se centran en preescolares, pues es sabido que intervenciones precoces obtienen mejores resultados que las intervenciones posteriores (Sepúlveda, 2012).

Por otro lado, los niños que cursan kínder son un grupo que no recibe mayor evaluación ni intervención desde el punto de vista psicomotor, lo que hace que estos niños queden relegados, por lo que las probabilidades de descubrir alteraciones en esta área se reducen enormemente (Monge, 1999).

En la revisión bibliográfica se encontraron estudios que relacionan Desarrollo Psicomotor y Nivel Socioeconómico (Sepúlveda, 2012). Se registró un mayor porcentaje de retraso y riesgo en el desarrollo psicomotor medido con la escala de Evaluación del Desarrollo Psicomotor (EEDP) y el Test de Desarrollo Psicomotor (TEPSI) y en el desarrollo emocional, en los grupos de nivel socioeconómico bajo.

En tal sentido, surge la necesidad de conocer y tener datos significativos acerca del Desarrollo Psicomotor de los niños con distintas situaciones de vulnerabilidad escolar que cursan kínder en los colegios municipalizados y particulares, ya que tener una referencia del comportamiento del niño(a) y su evolución en cada edad, significa poseer un instrumento para que tanto educadores como padres, puedan intervenir a tiempo en el desarrollo de los niños (González, 2001).

\section{MÉTODO}

Se utilizó un diseño no experimental, transversal y de acuerdo al nivel de profundidad, correlacional. El enfoque es de tipo cuantitativo/racionalista. 
Se procuró resguardar los siguientes criterios; de inclusión: niños de 4 a 5 años, que asisten a kínder en los establecimientos seleccionados, que se encuentren aptos físicamente para desarrollar las pruebas y cuyos padres firmaron el consentimiento informado; de exclusión: niños que se encuentren con licencia médica, niños con impedimento de realizar actividad física el día de la evaluación, niños con enfermedades neurológicas significativas, que puedan afectar la cognición.

Los niños fueron evaluados según los siguientes instrumentos: Escala de Desarrollo Perceptivo Motriz de Capón, 1981 - versión chilena (Carrasco, 1989 en Vargas, 2011). Este es un Test que evalúa el grado de desarrollo psicomotor en niños desde los 4 a 10 años de edad. Evalúa las áreas del conocimiento corporal, espacial, equilibrio, coordinación general y coordinación óculo-manual. Consta de una evaluación mínima de 6 puntos y máxima de
24 puntos que indicaría un muy buen desarrollo psicomotriz.

En este Test los niños deben realizar 6 pruebas distintas que miden el grado de ejecución motriz en las diferentes áreas: Tarea 1: evaluar el conocimiento de las partes del cuerpo y la coordinación motriz gruesa. Tarea 2: evaluación del equilibrio dinámico, lateralidad y asociación viso motriz. Tarea 3: evaluar la coordinación motriz gruesa, el equilibrio dinámico. Tarea 4: evaluar el equilibrio dinámico, la coordinación motriz gruesa y kinestésica. Tarea 5: evaluar orientación espacial y conciencia del cuerpo. Tarea 6: evaluar la coordinación ojomano y seguimiento con la vista. El evaluador registra en una planilla el puntaje obtenido del uno al cuatro, por los niños, en las seis pruebas y sumado el total de puntajes, los clasifica según los siguientes cinco baremos:

\begin{tabular}{ccccccc}
\hline \multicolumn{7}{c}{ CRITERIOS DE CLASIFICACIÓN } \\
\hline & \multicolumn{7}{c}{ MUY BUENO } & BUENO & ACEPTABLE & REGULAR & CON PROBLEMAS \\
\cline { 2 - 7 } & 10 o más & 24 & $23-22$ & $21-20$ & $19-18$ & 17 o menos \\
\cline { 2 - 7 } EDADES & $8-9$ & $24-23$ & $22-21$ & $20-19$ & $18-17$ & 16 o menos \\
\cline { 2 - 7 } & $7-6$ & $24-22$ & $21-19$ & $18-16$ & $15-13$ & 12 o menos \\
\cline { 2 - 7 } & $5-4$ & $24-22$ & $21-19$ & $18-15$ & $14-11$ & 10 o menos \\
\hline
\end{tabular}

Donde el puntaje mínimo a obtener son 6 puntos y el máximo son 24 puntos que corresponde al total del puntaje y el mejor desarrollo motriz posible.

Los colegios en los que se llevó a cabo el estudio fueron seleccionados según su dependencia económica. La escuela municipal, es un establecimiento urbano ubicado en la ciudad de Temuco. Según datos obtenidos a través del Ministerio de Educación, clasificada en el grupo socioeconómico medio-bajo, donde la mayoría de los apoderados han declarado tener entre 9 y 10 años de escolaridad y un ingreso del hogar que varía entre \$210.001 y \$340.000.

Entre el 59.01 y $78.00 \%$ de los estudiantes se encuentran en condición de vulnerabilidad social. El colegio particular en estudio es un establecimiento urbano ubicado en la ciudad de Temuco. Según datos obtenidos a través del Ministerio de Educación, clasificado en el grupo socioeconómico alto, la mayoría de los apoderados han declarado tener 16 o más años de escolaridad y un ingreso del hogar de $\$ 1.300 .001$ o más. El 0,00\% de los estudiantes se encuentran en condición de vulnerabilidad social (SIMCE, Ministerio de Educación, 2014). Se envió a ambos establecimientos una carta explicando el objetivo de las acciones a realizar, dónde firmaban aceptando su participación en la investigación. Los niños fueron evaluados en clases de educación física por los propios investigadores. Cada evaluación realizada de manera individual tomó 10 minutos. Concluida la evaluación con cada curso, se entregó una planilla con los resultados individuales de los test a la coordinación del área de Educación Física en cada establecimiento. 
El primer análisis fue de corte exploratorio para depurar la información, se procedió a determinar prevalencia de las principales variables de estudio en conjunto con un análisis descriptivo. Luego se determinó la normalidad de las variables a través de la prueba Kolmogórov-Smirnov. Para la comparación de las variables paramétricas cuantitativas entre dos grupos se utilizó el test $t$ de Student, y cuando existían más de dos, se realizó un ANOVA. En el caso de variables no paramétricas, se utilizó la prueba de la U de Mann-Whitney, cuando se comparaban dos grupos, y la prueba de la $\mathrm{H}$ de Kruskal-Wallis, para comparar más de dos grupos. Para la correlación de variables se utilizó la Rho de Spearman, debido al carácter no paramétrico. Todos los análisis se realizaron con el programa estadístico informático SPSS, versión 22.0. El nivel de confianza fue del 95\%, $(\mathrm{p}<0.05)$.

\section{RESULTADOS}

Los resultados obtenidos son expuestos en 5 tablas donde: la Tabla 1 da a conocer la comparación de las variables según tipo de establecimiento; la Tabla 2, comparación de variables según género; la Tabla 3 , comparación de variables según estado nutricional; la Tabla 4, correlación del peso con batería de test y la Tabla 5 se refiere a la frecuencias según criterios.

En la comparación de las variables, según tipo de establecimiento, los estudiantes del colegio particular, presentan diferencias significativas en todas las pruebas del test, frente a los resultados de la escuela municipal. Los estudiantes del colegio particular, presentan mayor desarrollo psicomotor obteniendo diferencias significativas en el total del test $(\mathrm{p}<0.05)$. El peso y la talla no presentaron diferencias significativas $(\mathrm{p}>0.05)$ (Tabla 1$)$.

Tabla 1

Comparación de variables según tipo establecimiento educacional.

\begin{tabular}{lccccccc}
\hline \multicolumn{1}{c}{ Variables } & \multicolumn{2}{c}{ Total $(\mathrm{n}=54)$} & Municipal $(\mathrm{n}=27)$ & \multicolumn{2}{c}{ Particular $(\mathrm{n}=27)$} \\
\hline Edad & 5.09 & 0.46 & 5.00 & 0.51 & 5.18 & 0.39 & Valor P \\
\hline Prueba 1 & 3.31 & 0.75 & 2.81 & 0.68 & 3.81 & 0.40 & 0.000 \\
\hline Prueba 2 & 3.33 & 0.82 & 2.78 & 0.80 & 3.89 & 0.32 & 0.000 \\
\hline Prueba 3 & 3.13 & 0.73 & 2.70 & 0.67 & 3.56 & 0.51 & 0.000 \\
\hline Prueba 4 & 3.23 & 0.70 & 2.77 & 0.59 & 3.67 & 0.48 & 0.000 \\
\hline Prueba 5 & 3.19 & 0.70 & 2.81 & 0.62 & 3.56 & 0.58 & 0.000 \\
\hline Prueba 6 & 3.50 & 0.64 & 3.26 & 0.71 & 3.74 & 0.45 & 0.007 \\
\hline Puntaje total & 19.63 & 3.25 & 17.04 & 1.99 & 22.22 & 1.91 & 0.000 \\
\hline \% de Logro & $81.79 \%$ & $13.15 \%$ & $79.99 \%$ & $8.29 \%$ & $92.59 \%$ & $7.95 \%$ & 0.000 \\
\hline Talla & 122.67 & 3.54 & 122.67 & 3.57 & 122.67 & 3.57 & 0.999 \\
\hline Peso & 23.43 & 3.11 & 24.00 & 3.37 & 22.85 & 2.76 & 0.158 \\
\hline
\end{tabular}

Los valores mostrados son media y desviación estándar, valores $p<0.05$ son estadísticamente significativos. Prueba 1: identificar partes del cuerpo. Prueba 2: tabla de equilibrio. Prueba 3: saltar con un pie tres veces. Prueba 4: salto y caída. Prueba 5: recorrido con obstáculos. Prueba 6: recepción de un balón. Estado nutricional.

Al comparar las variables según género, se aprecia que no existieron diferencias significativas en el rendimiento motor $(\mathrm{p}>0.05)$, solo la talla presentó diferencias estadísticamente significativas, con un valor superior en los hombres $(\mathrm{p}<0.05)($ Tabla 2$)$. 
Tabla 2

Comparación de variables según género.

\begin{tabular}{lccccc}
\multicolumn{1}{c}{ Variables } & \multicolumn{2}{c}{ Mujer $(\mathrm{n}=22)$} & \multicolumn{3}{c}{ Hombre $(\mathrm{n}=32)$} \\
\hline & Media & Desviación estándar & Media & Desviación estándar & Valor $\mathrm{P}$ \\
\hline Prueba 1 & 3.18 & 0.80 & 3.41 & 0.71 & 0.295 \\
\hline Prueba 2 & 3.05 & 0.95 & 3.53 & 0.67 & 0.052 \\
\hline Prueba 3 & 3.09 & 0.75 & 3.16 & 0.72 & 0.753 \\
\hline Prueba 4 & 3.05 & 0.58 & 3.35 & 0.75 & 0.071 \\
\hline Prueba 5 & 3.14 & 0.71 & 3.22 & 0.71 & 0.666 \\
\hline Prueba 6 & 3.45 & 0.60 & 3.53 & 0.67 & 0.501 \\
\hline Puntaje Total & 18.95 & 2.87 & 20.09 & 3.46 & 0.146 \\
\hline \% de Logro & 78.98 & 11.96 & 83.72 & 14.41 & 0.146 \\
\hline Talla & 121.59 & 3.85 & 123.41 & 3.16 & 0.040 \\
\hline Peso & 23.18 & 3.71 & 23.59 & 2.66 & 0.174 \\
\hline
\end{tabular}

Los valores mostrados son media y desviación estándar, valores $p<0.05$ son estadísticamente significativos. Prueba 1: identificar partes del cuerpo. Prueba 2: tabla de equilibrio. Prueba 3: saltar con un pie tres veces. Prueba 4: salto y caída. Prueba 5: recorrido con obstáculos. Prueba 6: recepción de un balón. Estado nutricional.

Al comparar las variables con el estado nutricional de los menores (Tabla 3), los sujetos con sobrepeso u obesidad, categorizados en malnutrición por exceso presentaron un rendimiento motor inferior en comparación con los normopeso y el grupo bajo peso; en las pruebas $1,2,4$ y 6 el puntaje total y el porcentaje de logro, presentaron diferencias significativas $(\mathrm{p}<0.05)$. Las pruebas 3,5 y 6 no alcanzaron significancia $(\mathrm{p}>0.05)$ (Tabla 3$)$.

\section{Tabla 3}

Comparación de variables según estado nutricional.

\begin{tabular}{lccccccc}
\hline \multicolumn{1}{c}{ Variables } & \multicolumn{2}{c}{ Bajo peso $(\mathrm{n}=6)$} & Normopeso $(\mathrm{n}=41)$ & Sobrepeso/obesidad $(\mathrm{n}=7)$ & Vediación \\
& Media & $\begin{array}{c}\text { Desviación } \\
\text { estándar }\end{array}$ & Media & $\begin{array}{c}\text { Desviación } \\
\text { estándar }\end{array}$ & Media & $\begin{array}{c}\text { Desviacíndar } \\
\text { estánd }\end{array}$ \\
\hline Prueba 1 & 3.67 & 0.82 & 3.37 & 0.70 & 2.71 & 0.76 & 0.045 \\
\hline Prueba 2 & 3.83 & 0.41 & 3.37 & 0.80 & 2.71 & 0.95 & 0.046 \\
\hline Prueba 3 & 3.50 & 0.84 & 3.15 & 0.73 & 2.71 & 0.49 & 0.121 \\
\hline Prueba 4 & 3.67 & 0.82 & 3.28 & 0.64 & 2.57 & 0.53 & 0.011 \\
\hline Prueba 5 & 3.33 & 0.82 & 3.17 & 0.74 & 3.14 & 0.38 & 0.805 \\
\hline Prueba 6 & 3.83 & 0.41 & 3.49 & 0.64 & 3.29 & 0.76 & 0.295 \\
\hline Puntaje Total & 21.83 & 3.92 & 19.73 & 3.12 & 17.14 & 1.86 & 0.014 \\
\hline \% de Logro & 90.97 & 16.33 & 82.22 & 13.01 & 71.43 & 7.77 & 0.014 \\
\hline Talla & 125.17 & 2.14 & 122.39 & 3.60 & 122.14 & 3.67 & 0.140 \\
\hline Peso & 21.00 & 0.89 & 22.83 & 1.91 & 29.00 & 3.96 & 0.000 \\
\hline
\end{tabular}

Los valores mostrados son media y desviación estándar, valores $p<0,05$ son estadísticamente significativos. Prueba 1; Identificar partes del cuerpo. Prueba 2; Tabla de equilibrio. Prueba 3; Saltar con un pie tres veces. Prueba 4; Salto y caída. Prueba 5; Recorrido con obstáculos. Prueba 6; Recepción de un balón. Estado nutricional 
Al aplicar la asociación de variables; peso y test. En la Prueba 1 presentó asociación negativa con el peso y de forma significativa $(\mathrm{p}=0.018)$, a mayor peso es menor el resultado en este Test. Las demás pruebas no presentaron asociación significativa ( $\mathrm{p}>0.05)$ (Tabla 4$)$.

Tabla 4

Correlación del Peso con batería de test.

\begin{tabular}{cccccccc}
\hline & Prueba 1 & Prueba 2 & Prueba 3 & Prueba 4 & Prueba 5 & Prueba 6 & Puntaje total \\
\hline Rho de Spearman & $-0.321^{*}$ & -0.166 & -0.105 & -0.215 & 0.023 & 0.034 & -0.181 \\
\hline Valor P & 0.018 & 0.230 & 0.448 & 0.122 & 0.871 & 0.808 & 0.189 \\
\hline
\end{tabular}

${ }^{*}$ La correlación es significativa en el nivel $p<0,05$.

Tabla 5

Frecuencias según criterios, expresado en \%.

\begin{tabular}{lcccccc}
\hline & \multicolumn{2}{c}{ Muestra Total } & \multicolumn{2}{c}{ Colegio Particular } & \multicolumn{2}{c}{ Colegio Municipal } \\
\hline & Frecuencia & $\%$ & Frecuencia & $\%$ & Frecuencia & Porcentaje \\
\hline Con problemas & 0 & $\%$ & 0 & $\%$ & 0 & $\%$ \\
\hline Regular & 3 & 5.6 & 0 & 0.0 & 3 & 11.1 \\
\hline Aceptable & 19 & 35.2 & 1 & 3.7 & 18 & 66.7 \\
\hline Bueno & 14 & 25.9 & 8 & 29.6 & 6 & 22.2 \\
\hline Muy bueno & 18 & 33.3 & 18 & 66.7 & 0 & 0.0 \\
\hline Total & 54 & 100.0 & 27 & 100.0 & 27 & 100.0 \\
\hline Datos presentados como frecuencia y porcentaje. &
\end{tabular}

Al evaluar las frecuencias según los baremos de clasificación del test, los menores de la escuela municipal concentraron sus resultados en el nivel aceptable. Y los estudiantes del colegio particular concentraron el porcentaje más alto de frecuencia en el nivel muy bueno.

\section{DISCUSIÓN}

A partir de los antecedentes recopilados y de la presente investigación, se pretendió conocer cómo afectan al desarrollo psicomotor aquellos factores que son indirectos, asociados también al ambiente del niño (Fernández y Riquelme, 2006).

En cuanto a la discusión de los resultados obtenidos, en primer lugar, se observó que en la comparación de los resultados del test y el tipo de establecimiento, los estudiantes del colegio particular, presentan diferencias significativas en todas las pruebas de estudio, frente a los resultados de la escuela municipal.
Los estudiantes del colegio particular presentan mayor rendimiento motor en las distintas pruebas del test. Con respecto al peso y la talla, no presentaron diferencias significativas. Esto confirma que los factores genéticos, la nutrición, la salud, el ámbito donde el niño crece y las oportunidades que le ofrece la familia son determinantes del desarrollo infantil. Un entorno familiar que fomenta el aprendizaje favorece el desarrollo integral del niño. Independientemente de las aptitudes que este posea para alcanzar altos niveles de competencia, si el medioambiente carente, la manifestación de sus capacidades puede verse comprometida (Garibottia y cols., 2013).

Al comparar las variables según su género, se aprecia que no existieron diferencias significativas en el rendimiento motor, solo la talla presentó diferencias, con un valor superior en los hombres. Esto concuerda con lo obtenido en la revisión bibliográfica, al investigar las diferencias de rendimiento físico entre niños y niñas, se indica que las diferencias biológicas 
son demasiado pequeñas y no pueden determinar tantas ventajas en los varones sobre las mujeres. En la niñez las aptitudes físicas son prácticamente iguales en ambos sexos (Losada, 2008).

Al comparar las variables con el estado nutricional de los menores, los sujetos con sobrepeso u obesidad, categorizados en malnutrición por exceso, presentaron un rendimiento motor inferior en comparación con los normopeso y el grupo bajo peso. Esto se debe a que los niños y adolescentes vienen practicando menos actividad física, convirtiéndose esta inactividad en una de las grandes causas del aumento del peso corporal y de la baja competencia motriz en los niños (López, Sotomayor, Álvarez, Céspedes, Poblete, Vásquez \& Escobar, 2009). Las recomendaciones de actividad física para la salud actualmente vigentes, exponen que todos los niños y adolescentes deberían participar en actividad de intensidad entre moderada y vigorosa, durante al menos una hora al día, y al menos dos veces por semana (Dos Santos, 2013).

Con respecto a la identificación de las partes del cuerpo, a mayor peso se lograron resultados inferiores. En este ámbito podemos inferir, según los resultados, que los preescolares de mayor peso, tienen mayores dificultades para poder reconocer su cuerpo, concordando con el juicio de expertos que plantean que los niños obesos tienen una pobre imagen corporal de sí mismos y expresan sensaciones de inferioridad, rechazo y baja autoestima (Martínez, Flores, Rizo, Aguilar, Vásquez \& Gutierrez, 2010).

Finalmente, observamos el desempeño motor obtenido en el test, con la siguiente clasificación porcentual de la muestra: el $0 \%$ de la muestra se clasificó con problemas; el 5.6\%, regular; el 35.2\%, aceptable; el $25.9 \%$, bueno y el $33.3 \%$ de la muestra como muy bueno. Por otra parte, los resultados del test obtenidos por cada colegio fue el siguiente: el $0 \%$ del la muestra del colegio particular fue clasificado como regular, en cambio el $11 \%$ de la muestra del colegio municipal se clasificó regular, solo un $3.7 \%$ de la muestra del colegio particular se clasificó como aceptable, en cambio un $66.7 \%$ de la muestra del colegio municipal se clasificó en aceptable; en el rango de clasificación bueno, el colegio particular obtuvo un $29.6 \%$ de su muestra a diferencia del colegio municipal que solo obtuvo un $22.2 \%$. Las diferencias se marcaron mucho más en el rango de clasificación muy bueno, donde los estudiantes del colegio particular obtuvieron un $66.7 \%$, mientras que los del colegio municipal obtuvieron un $0 \%$.

Según la evidencia expuesta en el párrafo anterior y lo contrastado con la revisión bibliográfica, se puede inferir que las diferencias en los resultados serían provocadas por el ambiente en que los preescolares en estudio se desarrollan cotidianamente. Las diferencias de estímulos recibidos desde el entorno familiar, social y comunitario, hacen que los preescolares tengan distintas experiencias que van formando y desarrollando sus potencialidades. A mayores estímulos recibidos, mayor desarrollo psicomotriz, por ende, a menor cantidad de estímulos, menor es el desarrollo psicomotriz (Seguel, 2012, Garibottia y cols., 2013).

\section{CONCLUSIÓN}

Los resultados indicaron que existen diferencias significativas en el desarrollo psicomotor entre los estudiantes del colegio particular y los pertenecientes a la escuela municipal. Así también, los sujetos con sobrepeso u obesidad, categorizados en malnutrición por exceso presentaron un rendimiento motor inferior en comparación con los normopeso y bajo peso. En el ámbito de la imagen corporal a mayor peso es menor el reconocimiento de la misma.

\section{REFERENCIAS BIBLIOGRÁFICAS}

Dos Santos, L. (2013). Desarrollo de las habilidades motoras fundamentales en función del sexo y del índice de masa corporal en escolares. Cuadernos de Psicología del Deporte, vol. 13, 63-72.

Fernández, L., Riquelme, P. (2006). Sistematización programa de estimulación temprana. Chile: Universidad de la Frontera/Universidad Católica de Temuco. 
Garibottia, G., Comar, H., Vasconi. C., Giannini, G. \& Pittau, C. (2013). Desarrollo psicomotor infantil y su relación con las características sociodemográficas y de estimulación familiar en niños de la ciudad de Bariloche, Argentina. Archivos Argentinos Pediatría, 111(5) 384390.

González, M. (2001). Pedagogía familiar. En: María del Luján González Tornaría, Pedagogía familar (pág. 162). Uruguay: Ediciones Trilce.

López, A., Sotomayor, L., Álvarez, M., Céspedes, P., Poblete, C., Vásquez, P., \& Escobar, M. (2009). Rendimiento Aeróbico en niños obesos de 6 a 10 Años. Revista chilena de pediatría, 80(5), 444-450. Recuperado en 06 de enero de 2016.

Losada, G. (2008). El salto de niñas y niños en edad escolar. La Aljaba Segunda Época, Volumen XII, 197-214.

Martínez-Aguilar, ML., Flores-Peña, Y., RizoBaeza, MM., Aguilar-Hernández, RM., Vásquez-Galindo, M. \& GutiérrezSánchez, G. (2010). Percepciones de la obesidad de adolescentes obesos estudiantes del $7^{\circ}$ al $9^{\circ}$ grado residentes en Tamaulipas, México. Revista Latinoamericana Enfermagem.[internet] 18(1): [07 pantalla]. Disponible en: http:// www.scielo.br/pdf/rlae/v18n1/es 08. pdf

Ministerio de Educación. (2014). Resultados SIMCE, 2014. Agencia de Calidad de la Educación. Disponible en: http://archivos.agenciaeducacion.cl/InformeTecnicoSimce_2014.pdf

Monge, M. (1999). Desarrollo psicomotor como elemento fundamental en el desarrollo integral de niños y niñas en edades tempranas. Educacion: Universidad de Costa Rica. Disponible en: https:// yessicr.files.wordpress.com/2013/03/ desarrollopsicomotordesarrollointegral.pdf
Quino, A. \& Barreto, P. (2015). Desarrollo motor en niños con desnutrición en Tunja, Boyacá. Revista Facultad Nacional de Salud Pública, 33(1) 15-21.

Schonhaut, L., Schönstedt, M., Álvarez, J., Salinas, P. \& Armijo, I. (2010). Desarrollo Psicomotor en Niños de Nivel Socioeconómico Medio-Alto. Revista chilena de pediatría, 81(2) 123- 128

Seguel, X. (2012). ¿Qué Efecto Tiene Asistir a Sala Cuna y Jardín Infantil Desde los Tres Meses Hasta los Cuatro Años de Edad? Estudio Longitudinal en la Junta Nacional de Jardines Infantiles: PSYKHE. http://www.scielo. cl/scielo.php?script $=$ sci_arttext\&pid $=\mathrm{S} 0718-22282012000200008$

Sepúlveda, G. (2012). Estudio comparativo entre el desarrollo psicomotor de niños que presentan o no vulnerabilidad escolar en primer año básico de colegios municipalizados y particulares subvencionados de la comuna de Iquique. Revista Motricidad y Persona, 10:17-24.

Vargas, C. (2011). Desarrollo motor: diseño, validación y propuesta de estimulación motriz. Alemania: Editorial Académica Española.

\section{Dirección para correspondencia}

Vanessa Carrasco Alarcón

Magíster en Educación Física

Departamento de Educación Física

Universidad de La Frontera- Chile

Contacto:

vanessa.carrasco@ufrontera.cl

Recibido: 20-05-2017

Aceptado: 08-09-2017 